\title{
Smooth Non-Abelian Bosonization
}

\author{
P.H. DAMGAARD \\ CERN - Geneva \\ and \\ R. SOllacher \\ Gesellschaft für Schwerionenforschung GSI mbH \\ P.O. Box 110552, D-64220 Darmstadt, Germany
}

May 6, 2019

\begin{abstract}
We present an extension of "smooth bosonization" to the non-Abelian case. We construct an enlarged theory containing both bosonic and fermionic fields which exhibits a local chiral gauge symmetry. A gauge fixing function depending on one real parameter allows us to interpolate smoothly between a purely fermionic and a purely bosonic representation. The procedure is, in the special case of bosonization, complementary to the approach based on duality.
\end{abstract}

CERN-TH-7347/94

hep-th/9407022

July 1994 


\section{Introduction}

Whereas (1+1)-dimensional bosonization of one fermion species, Abelian bosonization, has a long history (in field theory language most compactly expressed in the form given in ref. [1]), a proper bosonization of fermionic theories with internal symmetries - or just more species - was only achieved ten years ago [2]. The trouble is that if one naively applies the Abelian bosonization rules, one obtains a form of the action that is not manisfestly symmetric under the same non-Abelian transformations as the fermionic theory. Witten solved this problem in a very elegant way by showing that a free theory of $N$ Dirac fermions is equivalent to a bosonic $O(2 N) \times O(2 N)$-symmetric sigma model with a Wess-Zumino term [2]. A precursor of this equivalence can be found in the work of Polyakov and Wiegmann [3], and path-integral derivations of the non-Abelian fermi-bose equivalence in two dimensions have been given in ref. [4]. For a nice review, see also the textbook [5].

Surprisingly, Abelian bosonization can be been shown to be but a very special case within a huge class of equivalence [6]. This generalization of the conventional Abelian bosonization prescription is achieved by first finding a gauge-symmetric theory (with a particular chiral gauge symmetry that will be further explained below) containing both bosons and fermions. Two gauge fixings of this "higher" gauge-symmetric theory turn out to correspond to a description entirely in terms of fermions or entirely in terms of bosons. Bosonization (or fermionization) is thus nothing but a question of choosing a specific gauge in a particular gauge-symmetric theory of both bosons and fermions. More importantly, one can find a smooth gauge, parametrized in terms of one real variable $\Delta$ such that one moves smoothly from a fermion theory (reached at $\Delta=0$ ) to a boson theory (reached at $\Delta=1$ ) [6]. At all values of $\Delta$ in between, one has neither a purely fermionic nor purely bosonic description, but a mixed representation of apparently interacting bosons and fermions. All of these theories are equivalent. We have called this phenomenon smooth bosonization.

Very recently, Burgess and Quevedo [7] have shown that a natural generalization of duality transformations (as known in string theory or two-dimensional conformal field theory) in $(1+1)$-dimensional fermionic or bosonic theories can map one theory into the other. Their proposal for the appropriate generalization of the notion of duality is to take the route of Roček and Verlinde [8], and gauge a vector-like symmetry of the fermionic theory. After introducing a Lagrange multiplier to constrain one of the new degrees of freedom by fixing on flat connections, a genuine gauge can be chosen for the remaining single gauge degree of freedom. Upon integrating out the fermions and the gauge potential of the functional integral, the resulting theory of the Lagrange multiplier is precisely the appropriate bosonized theory. The bosonic and fermionic theories are thus, in this precise technical sense, really dual to each other. This approach is interesting, because it yields a complementary means of applying the same idea as in ref. [6]. It does not seem, however, to lead easily to the kind

of generalizations described in ref. [6]. In detail, the difference between the approach known as smooth bosonization and that based on duality can be described as follows. The duality transformation changes variables inside the functional integral by going from a fermionic field to a Lagrange multiplier, which ends up as the surviving bosonic field. The gauge degrees of freedom, as well as the original fermionic degrees of freedom, are explicitly integrated out of the functional integral. In contrast, smooth bosonization hinges on a change of variables in the functional integral, where the fermionic degrees of freedom are never integrated out 
of the path integral. Instead, they decouple in the "boson gauge" from the relevant Green functions. These ghostly remains of the fermions in the bosonic formulation are not entirely vanishing. They are fermions without currents (neither vector nor axial vector currents, one implying the other due to the two-dimensional identity $\gamma_{\mu} \gamma_{5}=-\epsilon_{\mu \nu} \gamma^{\nu}$, and hence have no charges. In any other gauge except this bosonic gauge they still carry non-trivial degrees of freedom. The bosonic degree of freedom in smooth bosonization is not the Lagrange multiplier of a gauge constraint, but rather the single gauge degree of freedom itself. Even in the particular case of the boson gauge $(\Delta=1)$, smooth bosonization is thus complementary to, and not identical to, the approach to bosonization based on duality.

Also non-Abelian bosonization can be given an interpretation in terms of duality transformations (see the second paper of ref. [7]). Here the notion of duality has to be somewhat enlarged to a non-Abelian context [9], but the procedure is otherwise the same. Again, the duality transformation does not appear to provide any new information on the fermi-bose equivalence, but it reproduces all known results. It is also the first time a step-by-step derivation of the appropriate non-Abelian bosonized theory, obtained directly by path-integral manipulations of the fermionic theory.

In view of these recent developments, the odds would seem to be in favour of a possible generalization of smooth bosonization to include the non-Abelian case. What we are seeking, then, is a gauge-symmetric theory of both fermions and bosons (with appropriate indices to make the action invariant also under global non-Abelian rotations). This theory should be constructed in such a way that a gauge exists which smoothly takes us from a fermionic theory to a bosonic theory as we vary a single parameter $\Delta$. This would give the sought-for generalization of non-Abelian bosonization. The aim of this paper is to provide an appropriate gauge-symmetric theory with these properties, and a smooth gauge which interpolates between fermionic and bosonic descriptions.

Such a smooth non-Abelian bosonization is of interest also from another point of view. Since both Abelian and non-Abelian bosonization can be thought of as obtained by applying duality transformations, the existence of a generalization of this notion to a continuous class of transformations whose endpoints coincide with the usual duality transformations (here fermi-bose transmutations) would strongly suggest that also the concept of duality in conformal field theory is open to generalization. As we will demonstrate here, it is indeed possible to generalize also non-Abelian bosonization to a smooth, larger, version. There thus seems to be increasing evidence that conventional duality transformations, be they Abelian or non-Abelian, may form only the endpoints of a much larger class of mappings that bring one theory into an equivalent one.]

Finally, obtaining the correct prescription for smooth non-Abelian bosonization in (1+1)dimensions is a much needed ingredient in defining suitable semi-bosonized versions of lowenergy effective Lagrangians in $(3+1)$ dimensions. Just as one can derive an effective longdistance action for the $\eta^{\prime}$ degree of freedom in QCD [12], one can apply the techniques of smooth non-Abelian bosonization to derive an effective quark-meson Lagrangian for the $S U\left(N_{f}\right)$ pseudoscalar multiplets of $N_{f}$-flavour QCD. But such a phenomenological application of our formalism, partial bosonization of regularized QCD, obviously lies much beyond the scope of the present paper.

\footnotetext{
${ }^{1}$ See also the discussion in section 2 of ref. [10].
} 


\section{Finding the gauge-symmetric theory}

The first step in establishing the existence of smooth non-Abelian bosonization consists in finding an appropriate gauge-symmetric theory of both bosons and fermions. Guided by the experience gained in the abelian case [6], we will derive this gauge-invariant theory by a collective field technique based on a local non-Abelian chiral rotation of fermions [11].

Our conventions are as follows. We consider $N$ species of Dirac fermions in Minkowski space:

$$
\psi=\left(\psi_{1}, \ldots, \psi_{N}\right) .
$$

The functional integral governing the dynamics of these fermions is chosen to be

$$
\begin{aligned}
Z[V, A]_{\Lambda} & =\int \mathcal{D}[\psi, \bar{\psi}]_{\Lambda} e^{i S_{\psi}} \\
\mathcal{S}_{\psi} & =\int d^{2} x \bar{\psi} i \not D \psi \\
D_{\mu} & =\partial_{\mu}-i V_{\mu}-i A_{\mu} \gamma_{5}
\end{aligned}
$$

Here, $V_{\mu}=V_{\mu}^{A} T_{A}$ and $A_{\mu}=A_{\mu}^{A} T_{A}$ are external sources; the $T_{A}$ 's are the generators of a Lie group $S U(N)$ or $U(N)$. By adding suitable terms depending only on these sources, and integrating over the sources in the functional integral, one can generate a number of nontrivial theories (non-Abelian Thirring models, non-Abelian gauge theories with fermions, etc.). So the generating functional (2) is sufficiently general for our purpose.[

In order to deal with a well-defined functional integral in (2) we must at least impose a regularization on the fermionic path integral, here indicated by the subscript $\Lambda$ which is the ultraviolet cut-off. We choose a consistent regularization scheme like the Pauli-Villars scheme described in ref. [13]. It explicitly preserves vector gauge symmetry, here non-Abelian phase rotations of the fermions. When mass terms are not present, one can in the end send the cut-off $\Lambda$ to infinity in a straightforward way (see below).

It is convenient to introduce projectors on definite chirality,

$$
P_{ \pm}=\frac{1}{2}\left(1 \pm \gamma_{5}\right)
$$

as well as light-cone components for an arbitrary two-vector $a_{\mu}$ :

$$
a^{ \pm}=\frac{1}{\sqrt{2}}\left(a^{0} \pm a^{1}\right)
$$

Using the relations

$$
\gamma^{+} P_{+}=\gamma^{+}, \gamma^{-} P_{-}=\gamma^{-}, \gamma^{+} P_{-}=0, \gamma^{-} P_{+}=0
$$

the action simplifies:

$$
\mathcal{S}_{\psi}=\int d^{2} x \bar{\psi} i\left(\gamma^{+} P_{+} D_{+}^{L}+\gamma^{-} P_{-} D_{-}^{R}\right) \psi
$$

\footnotetext{
${ }^{2}$ Mass terms lead to a far more complicated procedure, and will not be discussed in this paper. Compare also the different level of difficulty in deriving smooth Abelian bosonization with and without mass terms [6].
} 
Here we have defined

$$
D_{\mu}^{L}=\partial_{\mu}-i L_{\mu}=\partial_{\mu}-i V_{\mu}-i A_{\mu}
$$

and

$$
D_{\mu}^{R}=\partial_{\mu}-i R_{\mu}=\partial_{\mu}-i V_{\mu}+i A_{\mu}
$$

Only two couplings to external sources are actually playing a rôle, namely those to $L_{+}$and to $R_{-}$. This is the light-cone analogue of the relation $\gamma_{\mu} \gamma_{5}=-\epsilon_{\mu \nu} \gamma^{\nu}$ between $\gamma$-matrices in two dimensions.

Having fixed our notation, we now proceed by extending the system through the introduction of appropriate collective fields. We are of course guided by the experience gained in the Abelian case, where the appropriate field transformation involves a chiral rotation. In this case the natural procedure is to extend this to a non-Abelian chiral transformation. The bosonic fields are then matrices $U(x)$ being elements of a group $S U(N)$ or $U(N)$, i.e.,

$$
U(x)=e^{2 i \theta(x)} \quad, \quad \theta(x)=\theta^{A} T_{A}
$$

In the Abelian case [6] this extension of the field space was achieved by introducing a pseudoscalar field via a straightforward chiral transformation of the fermion fields. One can follow the same procedure in this non-Abelian case, but we choose for convenience to depart slightly from this route here, and introduce the fields $U(x)$ by a transformation involving only one chiral component of $\psi$ :

$$
\psi(x)=\left(U(x) P_{+}+P_{-}\right) \chi(x), \bar{\psi}(x)=\bar{\chi}(x)\left(U^{\dagger}(x) P_{-}+P_{+}\right)
$$

This transformation differs from a purely chiral rotation by an additional (non-Abelian) phase transformation $\chi(x) \rightarrow \exp (i \theta(x)) \chi(x)$.

It is well known that a chiral transformation like (10) has a non-trivial Jacobian due to the regularized fermionic measure in the functional integral. In ref. [13] one can find an expression for the Jacobian associated with this transformation (10):

$$
\begin{aligned}
\log J= & \int_{M} d^{2} x\left(\frac{1}{8 \pi} \operatorname{tr} \partial_{\mu} U \partial^{\mu} U^{\dagger}+\frac{i}{4 \pi} \operatorname{tr} U^{\dagger} L_{\mu} U\left(U^{\dagger} \partial^{\mu} U+\epsilon^{\mu \nu} U^{\dagger} \partial_{\nu} U\right)\right. \\
& \left.-\frac{i}{4 \pi} \operatorname{tr} R_{\mu}\left(U^{\dagger} \partial^{\mu} U-\epsilon^{\mu \nu} U^{\dagger} \partial_{\nu} U\right)-\frac{1}{4 \pi} \operatorname{tr}\left(R_{\mu}+\epsilon_{\mu \nu} R^{\nu}\right)\left(U^{\dagger} L^{\mu} U-L^{\mu}\right)\right) \\
& +\epsilon_{\mu \nu \rho} \frac{1}{12 \pi} \int_{B} d^{3} x \operatorname{tr} U_{s}^{\dagger} \partial^{\mu} U_{s} U_{s}^{\dagger} \partial^{\nu} U_{s}, U_{s}^{\dagger} \partial^{\rho} U_{s}+\mathcal{O}\left(\Lambda^{-2}\right) .
\end{aligned}
$$

The first integral is over 2-dimensional space-time, the second - the Wess-Zumino term - is over a 3-dimensional manifold with space-time $\mathrm{M}$ as its boundary. This expression corresponds to a local term in the new action, proportional to $\hbar$ if we re-instate factors of $\hbar$. In the limit where the ultraviolet cut-off is sent to infinity and in terms of light-cone coordinates, the result is simple:

$$
\begin{aligned}
Z[V, A]= & \int \mathcal{D}[\chi, \bar{\chi}] e^{i \int d^{2} x \mathcal{L}_{\chi}} \\
\mathcal{L}_{\chi}= & \bar{\chi} i\left(\gamma^{+} P_{+} D_{+}^{L, U}+\gamma^{-} P_{-} D_{-}^{R}\right) \chi+\frac{1}{4 \pi} \operatorname{tr} \partial_{+} U \partial_{-} U^{\dagger} \\
& +\frac{1}{4 \pi} \int_{0}^{1} d s \operatorname{tr} 2 i \theta\left[U_{s}^{\dagger} \partial_{+} U_{s}, U_{s}^{\dagger} \partial_{-} U_{s}\right]-\frac{i}{2 \pi} \operatorname{tr} L_{+} U \partial_{-} U^{\dagger}
\end{aligned}
$$




$$
-\frac{i}{2 \pi} \operatorname{tr} R_{-} U^{\dagger} \partial_{+} U-\frac{1}{2 \pi} \operatorname{tr} R_{-}\left(U^{\dagger} L_{+} U-L_{+}\right) .
$$

Here we have used the abbreviations

$$
U_{s}=e^{2 i s \theta}
$$

and

$$
D_{+}^{L, U}=\partial_{+}-i U^{\dagger} L_{+} U+U^{\dagger} \partial_{+} U
$$

For finite cut-off $\Lambda$, there are additional terms of order $\mathcal{O}\left(\Lambda^{-2}\right)$ which contain higher derivatives of $U(x)$, or higher powers of the external sources suppressed by suitable powers of the cutoff $\Lambda$. We will argue later that for the following one can neglect the effect of these terms in the limit of infinite cutoff.

Now we can extend the theory, treating the collective field $U(x)$ as a quantum field. This means that the functional integral (12) is averaged with respect to all possible configurations $U(x)$ :

$$
Z_{\text {ext }}[V, A]=\int \mathcal{D}[U] Z[V, A]
$$

As $Z[V, A]$ is independent of $U(x)$ such a manipulation just introduces an overall volume factor. Associated with this degeneracy is a local gauge symmetry. We choose to integrate $U(x)$ over the Haar measure. The partition function is then invariant under the local gauge transformation

$$
\begin{aligned}
\chi(x) & \rightarrow\left(A(x) P_{+}+P_{-}\right) \chi(x) \\
\bar{\chi}(x) & \rightarrow \bar{\chi}(x)\left(A^{\dagger}(x) P_{-}+P_{+}\right) \\
U(x) & \rightarrow U(x) A^{\dagger}(x) \\
U^{\dagger}(x) & \rightarrow A(x) U^{\dagger}(x)
\end{aligned}
$$

Here, $A(x)$ is a unitary matrix belonging to the same Lie group as $U(x)$. Note that neither the action nor the functional measure are separately invariant under this symmetry, but the combination is.

\section{A smooth gauge between fermions and bosons}

We have now achieved our first goal. We have found a new way of defining our fermionic theory so that it involves a non-trivial collection of bosonic fields as well. This means that we are now in a position to choose between different representations. It can be achieved by including a suitable $\delta$-functional inside the path integral — or in a different language, by choosing a gauge. We do it by imposing as many gauge constraints as there are new field degrees of freedom. In the present case this number is equal to the number of generators of the group, i.e. $N^{2}$ for $U(N)$ or $N^{2}-1$ for $S U(N)$.

Our aim is to provide a gauge which interpolates smoothly between a fermionic and a bosonic representation. Glancing at the transformed action in (12) one observes that this certainly can be achieved for at least one quantity, namely one of the currents coupling to the external sources. For example, by taking a derivative with respect to the source $L_{+}$, 
we find, inside the path integral, the following shift due to the introduction of the collective field $U(x)$ :

$$
\bar{\psi} \gamma^{+} P_{+} T_{A} \psi=\bar{\chi} \gamma^{+} P_{+} U^{\dagger} T_{A} U \chi-\frac{i}{2 \pi} \operatorname{tr} T_{A} U D_{-}^{R} U^{\dagger}
$$

Although the whole physical current - the left hand side of eq. (16) - is gauge invariant, each of the transformed components in eq. (17) are not. As in the Abelian case [6], we now try to choose a gauge such that the bosonic part describes a fraction $\Delta$ of the whole physical current. This is not entirely trivial, since the addition of the gauge-fixing constraint in itself modifies the regulator, which in turn, for consistency, modifies the Jacobian associated with the non-Abelian chiral transformation. In ref. [6] we called this phenomenon "anomalous gauge fixing", because in the Abelian case the pertinent gauge fixing turned out to hinge directly on the $U(1)$ anomaly in $(1+1)$ dimensions. Actually, the phenomenon is more general, and not necessarily linked to anomalies. It occurs whenever the gauge-fixing function causes modifications at the quantum level (here, at the one-loop level, because it occurs directly as a consequence of a non-trivial Jacobian, of order $\hbar$ when exponentiated into the action). So a more apt name would be "gauge fixing at the quantum level". In any case, for a situation similar to the present, we have already provided a fairly straightforward recipe in ref. [14]. The idea is to choose a more conventional gauge (in this case, entirely in the bosonic $U(x)$-sector) where no modification of the regulator is required. Since, on the other hand, this does not provide the gauge we are seeking, one more ingredient is needed. The way to move into the more interesting gauges from one of these trivial or "classical" ones, is to shift the external sources by the same Lagrange multiplier that enforces the gauge constraint. One can easily show, and it will be demonstrated explicitly below on the basis of BRST invarience, that this is a correct procedure.

Using a Lagrange multiplier field $b=b^{A} T_{A}$, we hence both shift the source $L_{+}$by the amount $\Delta b$ ( $\Delta$ being an arbitrary real number, the parameter which will turn out to smoothly join fermionic and bosonic gauges) and add a term

$$
\frac{i}{2 \pi} \operatorname{tr} b U D_{-}^{R} U^{\dagger}
$$

to the action.

This is not the full story, however. We find the associated Faddeev-Popov determinant by performing an infinitesimal gauge variation. Expressed in terms of conventional non-Abelian ghost fields, we get

$$
\mathcal{L}_{\text {ghost }}=\frac{1}{2 \pi} \operatorname{tr} U^{\dagger} \bar{c} U D_{-}^{R} c
$$

where $\bar{c}=\bar{c}^{A} T_{A}$ and $c=c^{A} T_{A}$ are the Grassmann-odd ghost fields (in the adjoint representation). Covariant derivatives acting on $c$ are correspondingly defined as

$$
D_{-}^{R} c=\partial_{-} c-i\left[R_{-}, c\right] \text {. }
$$

Similar relations hold for $U, b$ and $\bar{c}$.

With these ingredients the original functional integral now reads:

$$
Z[V, A]=\int \mathcal{D}[\chi, \bar{\chi}] \mathcal{D}[U] \mathcal{D}[b] \mathcal{D}[c, \bar{c}] e^{i \int d^{2} x \mathcal{L}_{\Delta}}
$$




$$
\begin{aligned}
\mathcal{L}_{\Delta}= & \bar{\chi} i\left(\gamma^{+} P_{+}\left(\partial_{+}-i U^{\dagger}\left(L_{+}+\Delta b\right) U+U^{\dagger} \partial_{+} U\right)+\gamma^{-} P_{-} D_{-}^{R}\right) \chi \\
& +\frac{1}{4 \pi} \operatorname{tr} \partial_{+} U \partial_{-} U^{\dagger}+\frac{1}{4 \pi} \int_{0}^{1} d s \operatorname{tr} 2 i \theta\left[U_{s}^{\dagger} \partial_{+} U_{s}, U_{s}^{\dagger} \partial_{-} U_{s}\right] \\
& -\frac{i}{2 \pi} \operatorname{tr}\left(L_{+}-(1-\Delta) b\right) U D_{-}^{R} U^{\dagger}-\frac{i}{2 \pi} \operatorname{tr} R_{-} U^{\dagger} \partial_{+} U \\
& +\frac{1}{2 \pi} \operatorname{tr} U^{\dagger} \bar{c} U D_{-}^{R} c .
\end{aligned}
$$

This functional integral is the desired representation of the theory, which interpolates between a purely fermionic and a purely bosonic formulation. We have derived it here using a slight variant of the Faddeev-Popov procedure, and since it involves a number of unusual ingredients it is worthwhile to confirm the derivation from a different point of view.

The idea is to use eq. (17) as the guide for gauge fixing. We wish to find a gauge such that the purely bosonic part of (17), i.e.

$$
-\frac{i}{2 \pi} \operatorname{tr}\left[T_{A} U D_{-}^{R} U^{\dagger}\right]
$$

carries a fraction $\Delta$ of the full physical current $\bar{\psi} \gamma^{+} P_{+} T_{A} \psi$. In the case of $\Delta=1$ this implies that the whole fermionic part of the rotated current vanishes entirely, i.e. that we are effectively inserting a $\delta$-function constraint setting this object to zero. Since this object, classically, is completely gauge invariant, an unusual phenomenon is clearly taking place here. The whole gauge-fixing procedure is, in this case $(\Delta=1)$, saved entirely by quantum effects: quantum mechanically $\bar{\chi} \gamma^{+} P_{+} U^{\dagger} T_{A} U \chi$ is not invariant under the gauge transformations (16). How can we in practice implement such a gauge? Consider the BRST procedure. Using the same ghosts, antighosts, and auxiliary fields as introduced in the Faddeev-Popov procedure discussed above, we first write down the corresponding BRST transformations:

$$
\begin{aligned}
\delta \chi(x) & =i c(x) P_{+} \chi(x) \\
\delta \bar{\chi}(x) & =-i \bar{\chi}(x) P_{-} c(x) \\
\delta U(x) & =-i U(x) c(x) \\
\delta U^{\dagger}(x) & =i c(x) U^{\dagger}(x) \\
\delta c(x) & =0 \\
\delta \bar{c}(x) & =b(x) \\
\delta b(x) & =0 .
\end{aligned}
$$

Next, we add to the un-fixed action a term of the form

$$
\delta\left[\frac{i}{2 \pi} \operatorname{tr}\left(\bar{c} U D_{-}^{R} U^{\dagger}\right)\right]=\frac{i}{2 \pi} \operatorname{trb} U D_{-}^{R} U^{\dagger}+\frac{1}{2 \pi} \operatorname{tr} \bar{c} U\left(D_{-}^{R} c\right) U^{\dagger}
$$

which is BRST-exact. This simply fixes, in a standard manner, the bosonic objects

$$
\operatorname{tr} T_{A} U D_{-}^{R} U^{\dagger}
$$

to zero. The action is now no longer locally gauge invariant, but only invariant under the global BRST transformations. However, we have not yet reached the gauge we are looking for. To remedy this, we shift everywhere the sources $L_{+}$by an amount $\Delta b, \Delta$ being the real 
parameter discussed above. In BRST formalism, such a procedure is certainly legal. First, what we have done by shifting the sources $L_{+}$in this manner preserves BRST symmetry. This is obvious, since the action is BRST invariant for arbitrary sources $L_{+}$, and $b(x)$ is itself inert under BRST transformations. Second, and equally important, this procedure does not change the regularization of the path integral. In general, the addition of terms involving couplings between the auxiliary field and the fermions, even when done in an apparently BRST-invariant manner, will lead to a mismatch between the functional measure and the action, and BRST invariance will be spoiled by terms of order $\hbar$. By shifting the source $L_{+}$ everywhere, BRST symmetry is guaranteed even at the quantum level." So this procedure provides a correct BRST gauge fixing of the path integral, and it is seen to coincide with the expression (20) above. The rest of this section is concerned with showing that it also provides precisely the smooth gauge that interpolates between fermions and bosons.

As the argument is presented above, it is far from obvious that what we have really reached is a gauge that fixes a certain fraction $\Delta$ of the physical non-Abelian current $\bar{\psi} \gamma^{+} P_{+} T_{A} \psi$ to be given by the bosonic current $(i / 2 \pi) \operatorname{tr} T_{A} U D_{-}^{R} U^{\dagger}$. To see that it is, we carry out explicitly the integration over the auxiliary field $b(x)$. This leads to $\delta$-functions inside the functional integral, implementing the constraints

$$
\Delta \bar{\chi} \gamma^{+} P_{+} U^{\dagger} T_{A} U \chi+(1-\Delta) \frac{i}{2 \pi} \operatorname{tr} T_{A} U D_{-}^{R} U^{\dagger}=0
$$

which, when comparing with eq. (17), is just what is required to ensure that the bosonic current is a fraction $\Delta$ of the full physical current.

The gauge above thus smoothly bosonizes the current $\bar{\psi} \gamma^{+} P_{+} T_{A} \psi$, but this is of course not the same as bosonizing the full theory. What about the currents coupled to $R_{-}$, and what about the coupling of $U$ to the ghost fields?

A number of miracles conspire to also:

- Decouple, after a field redefinition, the ghost term.

- Eliminate the coupling to the sources $R_{-}$in the "boson gauge" $\Delta=1$.

The key is vector current conservation. Let us first write the external sources as

$$
L_{+}=i l^{\dagger} \partial_{+} l, R_{-}=i r^{\dagger} \partial_{-} r .
$$

Unless $L_{+}$and $R_{-}$have some global topological properties f $^{\text {such }}$ rewritings are always possible.

\footnotetext{
${ }^{3}$ In the present case, where, in the infinite cut-off limit, the full action is at most linear in the sources $L_{+}$, the BRST derivation presented above may seem unnecessary, since the more simple Faddeev-Popov procedure is adequate. However, when keeping terms of order $1 / \Lambda^{2}$ in the expansion of the Jacobian (where $\Lambda$ is the overall ultraviolet cut-off), this more general BRST gauge-fixing procedure is by far superior. It is also the easiest path to derive the corresponding gauge-fixed action in higher dimensions.

${ }^{4}$ For example, in the $N$-flavour Schwinger model part of the Abelian component of the external vector source $V_{\mu}$ can be identified with the gauge field. As already noted in $[6]$, this field may carry topological charge, and hence cannot be expressed simply as $V_{\mu}=\epsilon_{\mu \nu} \partial^{\nu} \sigma+\partial_{\mu} \phi$. Instead a constant component can be added [6].
} 
Now we perform a set of unitary transformations, namely

$$
\begin{aligned}
& \bar{c} \rightarrow U r^{\dagger} \bar{c} r U^{\dagger} \\
& c \rightarrow r^{\dagger} c r
\end{aligned}
$$

which turn the Lagrangian $\mathcal{L}_{\Delta}$ in $(20)$ into

$$
\begin{aligned}
\mathcal{L}_{\Delta} \rightarrow & \bar{\chi} i\left(\gamma^{+} P_{+}\left(\partial_{+}-i \Delta U^{\dagger} b U+U^{\dagger} D_{+}^{L} U\right)+\gamma^{-} P_{-} D_{-}^{R}\right) \chi \\
& +\frac{1}{4 \pi} \operatorname{tr} \partial_{+} U \partial_{-} U^{\dagger}+\frac{1}{4 \pi} \int_{0}^{1} d s \operatorname{tr} 2 i \theta\left[U_{s}^{\dagger} \partial_{+} U_{s}, U_{s}^{\dagger} \partial_{-} U_{s}\right] \\
& -\frac{i}{2 \pi} \operatorname{tr}\left(L_{+}-(1-\Delta) b\right) U D_{-}^{R} U^{\dagger}-\frac{i}{2 \pi} \operatorname{tr} R_{-} U^{\dagger} \partial_{+} U \\
& \frac{1}{2 \pi} \operatorname{tr} \bar{c} \partial_{-} c .
\end{aligned}
$$

The ghosts have decoupled now.

The Lagrangian (26) may appear quite complicated, but in certain gauges this is deceptive. We will demonstrate below that in the two particular cases $\Delta=0$ and $\Delta=1$, one finds the required simplifications that were advertised above. We have already seen that in these two particular cases, the current $\bar{\psi} \gamma^{+} P_{+} T_{A} \psi$ is represented either entirely in terms of a fermionic current, or a bosonic current. We thus expect that in the gauge $\Delta=0$ we are dealing with a completely fermionic representation, and in the gauge $\Delta=1$ with a completely bosonic representation of the same theory. This is far from obvious, in both cases, since the gauge-fixing functions in this non-Abelian case remove entirely neither the fermionic fields, nor the bosonic fields.

Since all fields are always present in the path integral, the proof of fermionization $(\Delta=0)$ and bosonization $(\Delta=1)$ will rely on showing that in these two limiting cases we can decouple either the bosonic or the fermionic fields from the external sources $L_{+}$and $R_{-}$. The easiest way is to go back to the starting expression in terms of the action (6). Note that a pure non-Abelian phase rotation,

$$
\psi \rightarrow r^{\dagger} \psi, \bar{\psi} \rightarrow \bar{\psi} r
$$

eliminates the coupling to $R_{-}$at the expense of providing a modified source $\bar{L}_{+}$:

$$
L_{+} \rightarrow \bar{L}_{+} \equiv i r l^{\dagger} \partial_{+}\left(l r^{\dagger}\right)
$$

This means that as long as (24) is a valid substitution for the external sources, we can equally well work with just a source $\bar{L}_{+}$defined as above (replacing $L_{+}$in eq. (6)), and then set $R_{-}$ equal to zero.

With that in mind, let us first concentrate on the case $\Delta=0$. In this case the auxiliary field $b(x)$ only couples to the bosonic field $U(x)$. But the source $\bar{L}_{+}$still couples to the bosonic fields as well, so it may appear as if this is not yet the sought-for fermionic representation of the theory. Could this be true? We have introduced the collective fields $U(x)$ redundantly in the path integral, and have, in this particular gauge, put one simple gauge-fixing condition on these new fields. Clearly such a procedure cannot introduce physical couplings to the redundant fields $U(x)$. This means that the couplings between $\bar{L}_{+}$and $U(x)$ must be illusory, cancelling out whenever we compute physical quantities. 
To see that this indeed is the case, we first perform a phase rotation of the fermion fields:

$$
\chi \rightarrow U^{\dagger} \chi \quad, \quad \bar{\chi} \rightarrow \bar{\chi} U .
$$

The resulting couplings of $U(x)$ are then

$$
\frac{i}{2 \pi} \operatorname{tr}\left(b-\bar{L}_{+}\right) U \partial_{-} U^{\dagger}+i \bar{\chi} \gamma^{-} P_{-} U \partial_{-} U^{\dagger} \chi .
$$

At this point it is important that, at least for the Lie groups we are considering, quantities like $U \partial_{-} U^{\dagger}$ can be expressed as a linear combination of the generators $T_{A}$ of the Lie group. Then, obviously, a shift

$$
b^{A} \rightarrow b^{A}+\bar{L}_{+}^{A}-\frac{2 \pi}{\alpha} \bar{\chi} \gamma^{-} P_{-} T^{A} \chi,
$$

where $\alpha \delta_{A B}=\operatorname{tr} T_{A} T_{B}$, eliminates the couplings of $U(x)$ to both the external source $\bar{L}_{+}$, and to the fermions. The constant $\alpha$ is here defined through the relation $\operatorname{tr} T_{A} T_{B}=\alpha \delta_{A B}$. The resulting Lagrangian, after a transformation $\chi \rightarrow r \chi, \bar{\chi} \rightarrow \bar{\chi} r^{\dagger}$ is, on account of (24):

$$
\begin{aligned}
\mathcal{L}_{\Delta=0} \rightarrow & \bar{\chi} i\left(\gamma^{+} P_{+} D_{+}^{L}+\gamma^{-} P_{-} D_{-}^{R}\right) \chi \\
& +\frac{1}{4 \pi} \operatorname{tr} \partial_{+} U \partial_{-} U^{\dagger}+\frac{1}{4 \pi} \int_{0}^{1} d s \operatorname{tr} 2 i \theta\left[U_{s}^{\dagger} \partial_{+} U_{s}, U_{s}^{\dagger} \partial_{-} U_{s}\right] \\
& +\frac{i}{2 \pi} \operatorname{tr} b U \partial_{-} U^{\dagger}+\frac{1}{2 \pi} \operatorname{tr} \bar{c} \partial_{-} c .
\end{aligned}
$$

This shows explicitly that the Green functions in this gauge are given entirely by the fermionic functional integral. The integration over $b, U$ and the ghosts $\bar{c}, c$ simply yields an unimportant normalization factor.

For $\Delta=1$, the auxiliary field $b(x)$ only couples to the fermions. We may now shift $b$ by $i U D_{+}^{\bar{L}} U^{\dagger}$, thereby eliminating any coupling of the fermions to $U$ or the external sources. Here, again, we make use of the fact that $i U D_{+}^{\bar{L}} U^{\dagger}$ may be expressed as a linear combination of the generators $T_{A}$. In order to recover the original sources $L_{+}$and $R_{-}$again, we transform $U \rightarrow r U r^{\dagger}$ and use the definitions (24). The functional integration over $\chi, \bar{\chi}, b, c$ and $\bar{c}$ will then only yield a normalization factor $\mathcal{N}$ and the final result is a generating functional

$$
\begin{aligned}
Z[V, A]= & \mathcal{N} \int \mathcal{D}[U] e^{i \int d^{2} x \mathcal{L}_{\text {bos }}} \\
\mathcal{L}_{\text {bos }}= & +\frac{1}{4 \pi} \operatorname{tr} \partial_{+} U \partial_{-} U^{\dagger}+\frac{1}{4 \pi} \int_{0}^{1} d s \operatorname{tr} 2 i \theta\left[U_{s}^{\dagger} \partial_{+} U_{s}, U_{s}^{\dagger} \partial_{-} U_{s}\right] \\
& -\frac{i}{2 \pi} \operatorname{tr} L_{+} U D_{-}^{R} U^{\dagger}-\frac{i}{2 \pi} \operatorname{tr} R_{-} U^{\dagger} \partial_{+} U .
\end{aligned}
$$

This constitutes the desired bosonic representation of the original functional integral (2)). Note that, just as in the Abelian case, the fermions have not been gauged completely away in this formulation. They only happen to decouple from the external sources in this gauge, having neither (non-Abelian) vector nor axial vector currents. For this reason it is convenient to simply integrate them out, together with the ghosts and auxiliary fields.

By taking functional derivatives with respect to the external sources, we read off the effective bosonization rules from the representation (31):

$$
\bar{\psi} \gamma^{+} P_{+} T_{A} \psi \quad \sim \quad-\frac{i}{2 \pi} t r T_{A} U \partial_{-} U^{\dagger}
$$




$$
\bar{\psi} \gamma^{-} P_{-} T_{A} \psi \sim-\frac{i}{2 \pi} \operatorname{tr} T_{A} U^{\dagger} \partial_{+} U
$$

These bosonization rules, and the bosonized action (31), coincide with those derived by Witten [2]. When products of currents are taken, it follows from (31) that additional "contact terms", originating from the part involving $L_{+}$and $R_{-}$simultaneously, may arise. Also these terms, which depend on the regularization chosen, are well understood [4]. If one considers commutators of currents, the contact terms do not contribute, and the above simple current bosonization rules suffice.

For any other gauge with $\Delta \neq 1$, it is not possible to write down analogous bosonization relations. The theory is then only in its partially bosonized form, and there are both fermionic and bosonic contributions to physical Green functions. As in the Abelian case [6], one may even contemplate gauges for which $\Delta$ becomes a function of the space-time position as well. The existence of mixed representations which involve both fermions and bosons in a nontrivial interactive manner could not be inferred from the original bosonization arguments.

As in the Abelian case, it is straightforward to introduce genuine interaction terms in fermionic language, and use the method of smooth bosonization to find their bosonized or partly bosonized equivalents. This is done, for vector and axial vector interactions, by treating (part of) the sources $L_{+}$and $R_{-}$as dynamical degrees of freedom, to be integrated over in the functional integral with an appropriate measure. In this manner one can immediately treat two-dimensional thories such as the non-Abelian Thirring model and $N_{f}$-flavour QCD $_{2}$ (of an arbitrary number of colours $N_{c}$ ).

\section{Comments and conclusions}

Our first comment concerns the regularization of the original fermionic path integral, and the terms of order $\mathcal{O}\left(\Lambda^{-2}\right)$ in the effective action. The structure of these additional terms is similar to the Abelian case, and in both $(1+1)$ and $(3+1)$ dimensions [6, 12]. The higher derivatives acting on $U(x)$ can thus be interpreted as an induced "regularization" on these bosonic fields. Such a regularization is to be expected, because we started with a regularized functional integral and have performed manipulations which should not change this feature. The terms involving higher powers of the external sources are certainly suppressed in the limit $\Lambda \rightarrow \infty$, except perhaps for pathological cases where these sources are taken to be of the order of the cutoff. Even if these external sources are taken to be dynamical fields, this will not happen; any gauge theory in $(1+1)$ dimensions has an explicit scale set by the coupling constant $g$, and not by the ultraviolet cutoff $\Lambda$. It should be noted, however, that removing these extra terms in the formal limit $\Lambda \rightarrow \infty$ is not entirely as simple as it may appear on the surface, even here in $(1+1)$ dimensions. In particular, if mass terms are included the cut-off-dependent terms turn out to play a crucial rôle. See ref. [6] for a discussion of the corresponding Abelian case.

Another comment concerns the choice of gauge as implemented in (20) which smoothly interpolates between fermionic (at $\Delta=0$ ) and bosonic (at $\Delta=1$ ) descriptions. This gauge is well defined up to a zero mode, as is obvious if we consider the special case of $\Delta=0$ in the formulation in which the source $R_{-}$has been removed by a phase rotation (and then effectively included in the modified source $\bar{L}_{+}$). Here it clearly fixes only a combination 
involving the derivative of $U$ to zero : $\operatorname{tr} T_{A} U \partial_{-} U^{\dagger}=0$. $U$-component remains unfixed. For the massless case we have been considering here, this incompleteness in the chosen gauge fixing is of no concern, but it is crucial to gauge away this zero mode if one wishes to treat also the massive case correctly (see the second reference of [6]).

To conclude, we have provided one possible non-Abelian generalization of smooth bosonization. In the particular case of the bosonization gauge $\Delta=1$, we find an entirely bosonic theory based on a sigma model with a Wess-Zumino term, while in the fermionic gauge $\Delta=0$, we find a purely fermionic theory. By tracing the coupling to external sources $L_{+}$ and $R_{-}$, we regain the non-Abelian bosonization rules of Witten. For all other values of the gauge parameter $\Delta$ we obtain mixed representations of fermions and bosons. All of these theories are equivalent.

A natural question at this stage concerns the generalization of this bosonization prescription to higher dimensions. It should be obvious that a number of relations, very particular to two space-time dimensions, conspire to ensure the existence of a (local) purely bosonic gauge when a purely fermionic gauge exists. It is not inconceivable that three-dimensional bosonization based on the introduction of a Chern-Simons term [15] can be shown similarly to be part of a larger class of equivalence. In four dimensions the hopes for a local bosonic quantum field theory being entirely equivalent to a local fermionic theory are small. This does not imply that various stages of "partial bosonization" cannot be achieved. In fact, the present formalism is ideally suited for a formulation of theories with fermions (such as QCD) in partly bosonized terms. The gauge-fixing function then stipulates precisely which objects can be represented in bosonic variables. Some examples have been given in refs. [12, 14].

ACKNOWLEDGEMENT: We both acknowledge encouraging and stimulating discussions with Holger Bech Nielsen. One of us (P.H.D.) would also like to thank Cliff Burgess and Fernando Quevedo for discussions. We finally wish to thank D.M. Brink for giving us the opportunity to enjoy a pleasant stay at the European Centre for Theoretical Studies in Nuclear Physics and Related Areas, ECT* ${ }^{*}$, in Trento, Italy, where the work described here was completed. This work was also supported in part by Norfa grant no. 93.15.078/00.

\section{References}

[1] S. Coleman, Phys. rev. D11 (1975) 2088.

S. Mandelstam, Phys. Rev. D11 (1975) 3026.

A. Casher, J. Kogut and L. Susskind, Phys. Rev. D10 (1974) 732.

[2] E. Witten, Commun. Math. Phys. 92 (1984) 455.

[3] A.M. Polyakov and P.B. Wiegmann, Phys. Lett. 141B (1984) 223.

\footnotetext{
${ }^{5}$ The zero mode is in fact present for all values of $\Delta$. See the analogous discussion for the Abelian case in ref. [6].
} 
[4] P. DiVecchia, B. Durhuus and J.L. Petersen, Phys. Lett. 144B (1984) 245.

D. Gonzales and A.N. Redlich, Phys. Lett. 147B (1984) 150.

P. DiVecchia and P. Rossi, Phys. Lett. 140B (1984) 344.

[5] E. Abdalla, M.C.B. Abdalla and K. Rothe: Non-perturbative Methods in 2-Dimensional Quantum Field Theory, World Scientific (Singapore) 1991.

[6] P.H. Damgaard, H.B. Nielsen and R. Sollacher, Nucl. Phys. B385 (1992) 227; Phys. Lett. B296 (1992) 132.

[7] C.F. Burgess and F. Quevedo, preprints hep-th/9401105 (to appear in Nucl. Phys. B) and hep-th/9403173.

[8] M. Roček and E. Verlinde, Nucl. Phys. B373 (1992) 630.

[9] X. de la Ossa and F. Quevedo, Nucl. Phys. B403 (1993) 377.

[10] E. Álvarez, L. Álvarez-Gaumé and Y. Lozano, preprint CERN-TH-7204/94, hep-th/9403155.

[11] J. Alfaro and P.H. Damgaard, Ann. Phys. (N.Y.) 202 (1990) 398.

[12] P.H. Damgaard, H.B. Nielsen and R. Sollacher, Nucl. Phys. B414 (1994) 541.

[13] R.D. Ball, Phys. Rep. 182 (1989) 1.

[14] P.H. Damgaard and R. Sollacher, Phys. Lett. B322 (1994) 131.

[15] M. Lüscher, Nucl. Phys. B326 (1989) 557. 